\title{
Iustina Radu
}

Doctorante, Académie des Études Économiques de Bucarest, Roumanie

(2011)

\section{"Le management des institutions sociales - choisir rationnellement} ne signifie pas choisir entre les crises et les préférences personnelles, mais choisir entre les institutions."

Un document produit en version numérique par Jean-Marie Tremblay, bénévole, professeur de sociologie retraité du Cégep de Chicoutimi

Courriel: jean-marie_tremblay@uqac.ca

Site web pédagogique: http://www.uqac.ca/jmt-sociologue/

Dans le cadre de: "Les classiques des sciences sociales" Une bibliothèque numérique fondée et dirigée par Jean-Marie Tremblay, professeur de sociologie au Cégep de Chicoutimi

Site web: $\underline{h t+p: / / c l a s s i q u e s . u q a c . c a /}$

Une collection développée en collaboration avec la Bibliothèque Paul-Émile-Boulet de l'Université du Québec à Chicoutimi Site web: http://bibliotheque.ugac.ca/ 


\section{Politique d'utilisation de la bibliothèque des Classiques}

Toute reproduction et rediffusion de nos fichiers est interdite, même avec la mention de leur provenance, sans l'autorisation formelle, écrite, du fondateur des Classiques des sciences sociales, Jean-Marie Tremblay, sociologue.

Les fichiers des Classiques des sciences sociales ne peuvent sans autorisation formelle:

- être hébergés (en fichier ou page web, en totalité ou en partie) sur un serveur autre que celui des Classiques.

- servir de base de travail à un autre fichier modifié ensuite par tout autre moyen (couleur, police, mise en page, extraits, support, etc...),

Les fichiers (.html, .doc, .pdf, .rtf, .jpg, .gif) disponibles sur le site Les Classiques des sciences sociales sont la propriété des Classiques des sciences sociales, un organisme à but non lucratif composé exclusivement de bénévoles.

Ils sont disponibles pour une utilisation intellectuelle et personnelle et, en aucun cas, commerciale. Toute utilisation à des fins commerciales des fichiers sur ce site est strictement interdite et toute rediffusion est également strictement interdite.

L'accès à notre travail est libre et gratuit à tous les utilisateurs. C'est notre mission.

Jean-Marie Tremblay, sociologue

Fondateur et Président-directeur général, LES CLASSIQUES DES SCIENCES SOCIALES. 
Cette édition électronique a été réalisée par Jean-Marie Tremblay, bénévole, professeur de sociologie au Cégep de Chicoutimi à partir de:

\section{Iustina Radu}

"Le management des institutions sociales - choisir rationnellement ne signifie pas choisir entre les crises et les préférences personnelles, mais choisir entre les institutions".

Texte inédit pour diffusion originale dans Les Classiques des sciences sociales comme préalable au dépôt de la thèse de doctorat, sous la direction du Professeur Ioan Radu, président de la Chaire de Gestion de l'Information, Académie des Études Économiques de Bucarest, Roumanie.

[Autorisation formelle accordée par l'auteur le 27 septembre 2008 de diffuser toutes ses publications dans Les Classiques des sciences sociales.]

Courriels : Iustina Radu : justinaradu@yahoo.com

Professeur Ioan Radu, ASE, Bucarest, Roumanie, directeur de thèse: iradu13@gmail.com

Polices de caractères utilisée : Comic Sans, 10 points.

Édition électronique réalisée avec le traitement de textes Microsoft Word 2008 pour Macintosh.

Mise en page sur papier format : LETTRE US, $\left.8.5^{\prime \prime} \times 11^{\prime \prime}\right)$

Édition numérique réalisée le 19 mai 2011 à Chicoutimi, Ville de Saguenay, Québec. 


\section{Iustina Radu}

Doctorante, Académie des Études Économiques de Bucarest, Roumanie

"Le management des institutions sociales - choisir rationnellement ne signifie pas choisir entre les crises et les préférences personnelles, mais choisir entre les institutions".

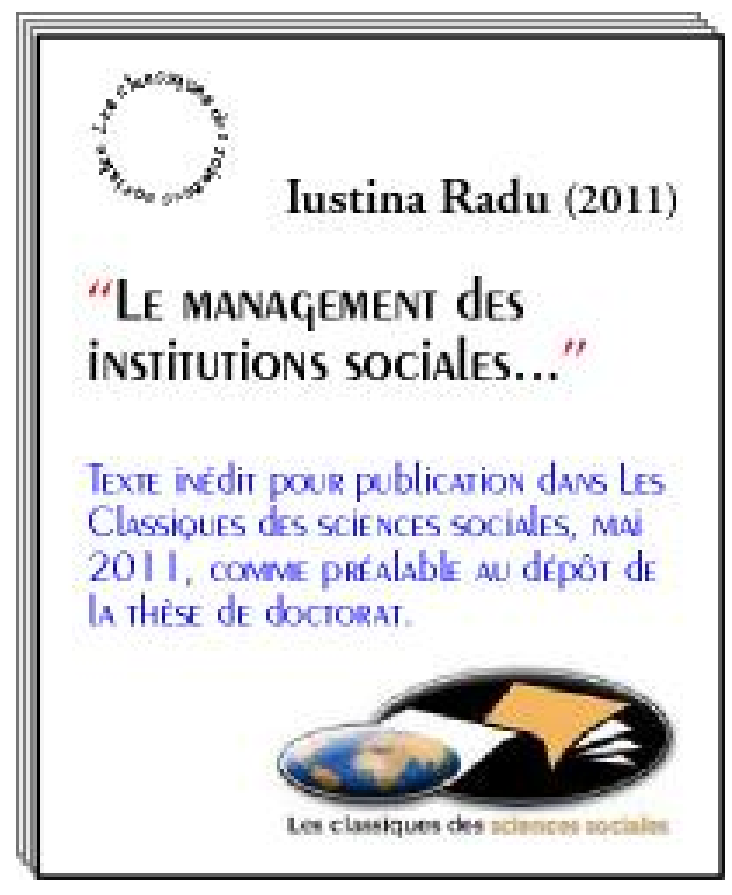

Texte inédit pour diffusion originale dans Les Classiques des sciences sociales comme préalable au dépôt de la thèse de doctorat, sous la direction du Professeur Ioan Radu, président de la Chaire de Gestion de l'Information, Académie des Études Économiques de Bucarest, Roumanie. 


\section{Table des matières}

Abstract

Bref historique ou le besoin de contrôle

L'évaluation de l'état de fait

Références au besoin de management du changement dans les institutions publiques du domaine social de Roumanie

Qu'est-ce que les citoyens veulent et qu'est-ce qu'ils reçoivent dans les institutions publiques

Quels instruments du domaine privé peuvent être utiles au secteur public

La stratégie de marketing de la nouvelle Inspection Sociale

Principe 1. la concentration sur les citoyens

Principe 2. la segmentation et le choix des piste cible

Principe 3. I'identification de la concurrence

Principe 4. I'utilisation des 4 instruments du mixe de marketing - le produit, le prix, la distribution, la promotion

Principe 5. la suite de l'activité et l'ajustement

La création de l'Inspection Sociale de la Roumanie

Bibliographie 


\section{Iustina Radu}

Doctorante, Académie des Études Économiques de Bucarest, Roumanie

Directrice exécutive

Consiliul National al Dizabilitatii din Romania (CNDR)

Strada Cauzasi nr. 26, Sector 3, Bucuresti, Romania

http://cndr.anvr.ro/

"Le management des institutions sociales - choisir rationnellement ne signifie pas choisir entre les crises et les préférences personnelles, mais choisir entre les institutions".

Texte inédit pour diffusion originale dans Les Classiques des sciences sociales comme préalable au dépôt de la thèse de doctorat, sous la direction du Dr Ioan Radu, professeur d'université et président de la Chaire de Gestion de l'Information, Académie des Études Économiques de Bucarest, Roumanie.

\section{ABSTRACT}

\section{Retour à la table des matières}

La différence entre les politiques sociales et les politiques publiques renvoie beaucoup de controverses en Roumanie. Pendant que les politiques sociales gèrent une intervention gouvernementale pour résoudre les problèmes sociaux, les politiques publiques peuvent être proactives.

D'habitude, les gens laissent les institutions prendre les décisions. La Roumanie de l'année 2011 se trouve indubitable dans une situation de crise sociale. Une crise majeure ne détruit pas les normes sociales. Dans une telle situation, la communauté quitte les principes moraux et va prendre les décisions d'urgence. La protection de ceux qui se trouvent dans les hautes positions garde la structure des institutions fondamentales et soutient les canaux de communication en fonction. Le résultat : le maintien d'un niveau minimum du fonctionnement.

Le changement du modèle de management des institutions publiques est inévitable et il va donner des résultats pour les citoyens et pour le secteur privé. Personne ne peut garantir l'existence où le niveau du financement des institutions publiques. Il faut que les institutions apprennent la dynamique des technologies en changement. Chaque institution publique doit avoir une conception stratégique, des nouvelle pratiques, efficientes, se renouveler, savoir présenter ses problèmes au grand public, par des stratégies d'image bien faite. 


\section{Bref historique ou le besoin de contrôle}

\section{Retour à la table des matières}

Jusqu'en 1990, les notions de «personne défavorisée» ou «personne avec un handicap», même si elles existaient dans le dictionnaire explicatif de la langue roumaine, n'avaient pas de correspondance dans la vie réelle. Jusqu'alors, la Roumanie était un pays dont les habitants n'avaient pas de problèmes, de besoins spéciaux, etc. En 1990 a été créé la première structure gouvernementale au cadre de l'exécutif, respectivement, Le secrétariat d'État pour les Handicapés, avec une représentation départementale dans le territoire qui devait coordonner méthodologiquement le domaine spécial des personnes avec handicap et qui élaborait le cadre légal en vue de l'évaluation et du contrôle du système social. Jusqu'en 2000, la direction du système était centralisée excessivement, tant concernant le financement des institutions social - éducatives, à l'embauchage et à la nominalisation du personnel du domaine social tout entier ainsi qu'aux prestations de services. En 2001, commence une période de décentralisation, brusque, sans avoir à la base une consolidation concernant l'évaluation du système, on déléguait les compétences à la subordination des centres sociaux aux autorités locales et départementales /chose méritoire/, mais sans préparer le personnel qui allait gérer une problématique si sensible et en même temps compliquée (chose ignorée par l'exécutif!). Le système se révolte d'une partie et de l'autre de la barricade, donc c'est ainsi que les personnes avec handicap, qui ne savent à qui ils peuvent s'adresser pour recevoir les droits, ainsi que les structures de l'administration centrale et locale, qui n'ont pas l'expertise et qui ne connaissent pas les limites et les compétences. Par la voix du secrétaire général du gouvernement de cette date là, l'exécutif, au lieu de gérer lucidement la situation de crise, menace au niveau de l'année 2002, qu'il va annuler ce domaine (pour le gouvernement prioritaire étant seulement le domaine des enfants, coordonné par une structure qui s'est modelée beaucoup plus rapidement sur les « requises de l'Europe», respectivement L'Autorité pour la protection des Droits de l'Enfant et Adoption). Suite aux problèmes très graves déclenchés par le changement, la nouvelle créée, L'Autorité Nationale pour les Personnes avec handicap, avec une représentation sur 8 régions, respectivement 8 Inspections Régionales, modifie totalement l'activité ainsi que le rôle de coordonnateur méthodologique, en se transformant, sans le cadre légal très bien 
délimité, dans la seule institution qui contrôlait et appliquait des sanctions à ceux qui géraient le domaine social.

Ce sont seulement deux actions majeures qui ont prouvé leur opportunité et qui ont tiré, d'une certaine façon, le système de l'impasse:

* L'évaluation de l'état de fait du domaine social (2002)-étude socio- psychologique au niveau national ${ }^{1}$, et

* La création du personnel dans les Inspections Régionales (20022004) 23

En 2005 c'est une autre syncope qui apparaît: I'annulation des inspections régionales, respectivement l'annulation du contrôle et la recentralisation du système au niveau de l'exécutif. Depuis septembre 2005 jusqu'au présent, la manque de l'action de contrôle mène à l'incohérence de l'application des politiques sociales, de l'impuissance d'attirer des fonds européennes et a l'incapacité de gérer une base unitaire et réelle de données des services et des prestations sociales en Roumanie. A la pression de l'Union Européenne a été créé L'inspection sociale par l'ordonnance d'Urgence du Gouvernement no. 130/2006 du 30 décembre 2006 approuvée par la Loi no.211/2007, institution qui est dans la subordination du Ministère du Travail, de la Famille et de l'Egalité des Chances, qui a comme but le contrôle de l'implémentation de la législation dans le domaine, ainsi que l'inspection de l'activité des institutions publiques et privées, responsables de la livraison des prestations et des services sociales. L'Inspection Sociale se lance officiellement en janvier 2008.

C'est pour le bon fonctionnement de l'Inspection Sociale qu'il faut faire une diagnose systématique qui a à la base trois requises:

1 «L'évaluation de l'état de fait du domaine social-2002 »-projet national déroulé par l'inspection Régionale Bucarest (dirigent projet Iustina Radu, inspecteur en chef de I.R. Bucarest)

2 «L'élaboration du standard occupationnel de l'assistant personnel de la personne avec handicap-2003»-projet déroulé par l'inspection régionale Bucarest en partenariat avec l'Institut National de Recherche concernant Le Combat de l'Exclusion Sociale (Iustina Radu - membre dans l'équipe de coordination et implémentation du projet)

3 «La formation de l'assistant personnel de la personne avec handicap-2004»projet déroulé par L'Inspection Régionale Bucarest en partenariat avec le Centre National de Ressources Juridiques ( Iustina Radu-chargée de cours/formateur) 
1. Ceux qui font la diagnose sur les problèmes et le développement de l'institution doivent bien connaître l'organisation et avoir une compréhension profonde des phénomènes (le changement en organisations est déterminée par les forces internes propres: d'une manière égale, l'organisation est influencée aussi par ce qui se passe outre elle, procès que le management ne peut pas influencer, elle se trouvant dans une dynamique continue qu'il ne peut pas influencer).

2. La diagnose doit décrire et interpréter l'organisation le plus exactement possible.

3. La diagnose doit offrir des motifs pour implémenter des plans d'actions.

\section{L'évaluation de l'état de fait}

\section{Retour à la table des matières}

Normalement, les individus remettent la prise des décisions aux institutions. La Roumanie de l'année 2011 se trouve dans une situation de crise sociale. Une crise majeure ne provoque pas une destruction des normes sociales. Dans une telle situation, la communauté passe du set habituel de principes moraux à un autre set habituel, celui d'urgence. Le système d'urgence ne signifie pas une abrogation de tous les principes, une chute des convictions, mais une diminution et une contrainte des principes normales de distribution. La protection de ceux qui sont à la direction garde la structure des institutions fondamentales et tient en état de fonctionnement les canaux de communications habituelles.

Le résultat est le maintient d'un niveau minime de fonctionnement. A mesure que la crise s'accentue on observe la destruction systématique de certaines catégories sociales. On peut prévoir qui est condamné à mort. Quelques soient les principes normatifs d'exclusion des privilèges ou de la sûreté offerte par la communauté, les exclusions légiférées établissent qui recevra de plus en plus moins-en même temps avec la diminution des ressources - et finalement, qui va rester en dehors ou qui va être laissé mourir de faim.

Il est surprenant car les recherches montrent que les victimes préétablies acceptent le sort avec résignation. Elles acceptent que le malheur qui leur est arrivé, pour eux et leurs familles, a été mérité, et qu'il faisait partie des conditions normales pendant une crise. Ils comprennent que les élites n'ont été aucun moment en 
péril et ils prennent dès le début les anciens rapports de servitude, sans souffrir, même avec reconnaissance. L'acceptation de la condition de victime montre qu'il ne s'agit pas d'une chute de l'ordre social, mais même de son affirmation. Les peuples ne raisonnent pas par l'intermède de leurs institutions, ce sont les institutions qui raisonnent pour eux (la Théorie de William Torry).

Une collectivité peut imposer à ses victimes préétablies de mener les difficultés en temps de crise et elle peut résoudre le problème des décisions de distribution, laissant les institutions faire le choix, mais seulement alors quand la communauté leur a conféré la légitimité. La distribution par l'intermède des institutions publiques responsables et compétentes est insatisfaisante, mais c'est le prix qui doit être payé, parce que nous vivons dans une société pluraliste, où la légitimité est toujours mise en doute.

Lorsque les individus se contredisent sur le thème de la raison élémentaire, le conflit le plus difficile à résoudre est celui entre les institutions fondées sur des principes incompatibles. Les obstacles contre la violence conjugale et contre les abus à l'égard des mineurs, contre les abus de l'alcool ou des drogues, contre le racisme ou la discrimination sexuelle n'ont aucun succès. Seulement la transformation des institutions peut changer les choses en bien. Il faut que nous nous y adressions, et non aux individus. Et il faut le faire en permanence, et non seulement en périodes de crise.

\section{Références au besoin de management du changement dans les institutions publiques du domaine social de Roumanie}

Retour à la table des matières

Il faut savoir le fait que les institutions légitimées prennent les décisions importantes, et les citoyens, quand ils doivent prendre des décisions importantes, ne peuvent pas le faire que dans les cadres délimités par les institutions qu'ils bâtissent eux-mêmes.

En Roumanie les institutions sont dépendantes du facteur politique, qui dirige les ressources publiques plusieurs fois en conformité aux intérêts de la clientèle.

Les citoyens trouvent avec grande difficulté leur place dans une société où les institutions perdent leur légitimité à cause du fait qu'elles sont remorquées aux intérêts de la clientèle politique qui se trouve en fonctions. Les décisions majeures sont 
prises en s'assumant la responsabilité gouvernementale, et les lois organiques sont détournées par des ordonnances d'urgence ${ }^{4}$. On a besoin de la formulation graduelle d'un réseau de concepts et de modèles articulés entre eux et, en même temps, le développement des organisations sociales adéquates. Les institutions seront de la même importance et elles devront être conscientes de l'existence de tous les autres partenaires sociaux et de coopérer l'une avec l'autre. Il faut aborder une vision systémique sur le monde du point de vue des relations et de l'intégration. Les systèmes sont des entités intégrés, dont les propriétés ne peuvent pas être réduites aux propriétés des parties composantes plus petites. Même si on peut discerner les parties individuelles qui composent un système, l'entier est toujours différent de la simple somme de ses parties composantes.

Conclusion: Le système de management devrait être un système écologiste - les systèmes naturelles sont autoréglables. Les institutions sont des organismes vivantes, qui raisonnent et examinent le degré dans lequel la pensée individuelle en est dépendante, ainsi que la manière dans laquelle les individus réussissent être d'accord sur le renoncement à l'intérêt personnel dans la faveur de la constitution des biens collectifs. La relation individu - institution va dans les deux directions, dans le but de la légitimité et de l'accord d'identité. C'est de la légitimité que les institutions sociales de la Roumanie d'aujourd'hui ont besoin.

\section{Qu'est-ce que les citoyens veulent et qu'est-ce qu'ils reçoivent dans les institutions publiques}

\section{Retour à la table des matières}

Toute société a besoin d'un secteur public, dont le principal rôle est celui de définir les principes de fonctionnement de la société.

Le deuxième rôle des institutions publiques est celui d'assurer le bon fonctionnement des services publics d'une importance vitale pour les citoyens. C'est ainsi que l'amélioration de l'activité des institutions publiques peut être réalisée par l'adaptation des instruments que le secteur privé utilise, pour développer plus efficacement son activité.

4 ex. O.G. no. 51/2005 par laquelle on modifie une loi spéciale, la Loi no.239/2003. 
Le troisième rôle est celui d'assurer les services publics que ni le secteur privé, ni le secteur non lucratif, ne veulent ou ne peuvent pas offrir avec les ressources existantes. Donc, l'administration offre, d'habitude, de l'assistance aux pauvres, indépendamment ou avec les institutions non gouvernementales.

\section{Quels instruments du domaine privé peuvent être utiles au secteur public}

\section{Retour à la table des matières}

C'est le perfectionnement continu de l'employé public, la participation aux cours, séminaires etc. qui sont importants pour être tout le temps au courant avec les nouveautés du domaine, pour ne pas dépendre de la fonction ou de l'ancienneté dans la fonction pour avancer. Les domaines à étudier :

* Le management de la qualité des processus

* Les stratégies basées sur les besoins des clients

* La restructuration

* Le mesurage et l'évaluation de l'activité

* Les systèmes de simulation du management

* Les analyses coût - bénéfice et coût efficacité

* L'administration électronique et information électronique

* Les organisations basées sur les connaissances etc.

L'appel à l'efficacité et le renouvellement des institutions gouvernementales semble, dans la Roumanie d'aujourd'hui, en regardant de dehors, une chose irréaliste. Pourtant, les institutions publiques sont soumises aux nouvelles provocations et aux actions entreprises par les nouveaux concurrents, n'importe quelles d'entre elles pouvant imposer une restructuration, une réorganisation ou même la disparition de l'organisation. De même comme les firmes privées, les institutions publiques doivent comprendre la dynamique des forces et des technologies qui sont en changement: elles doivent avoir une conception stratégique; ils doivent apporter de nouvelles pratiques: innover ; elles doivent savoir présenter les mérites du public en général et aux catégories de public qui payent des impôts et qui suivent leur activité. 


\section{La stratégie de marketing de la nouvelle Inspection Sociale}

Retour à la table des matières

La stratégie de marketing se manifeste dans l'évolution et la transformation continue d'une institution, qui a à la base la connaissance des principes fondamentaux et elle est cultivée par la pratique, des feedback et des ajustassions.

\section{Principe 1 - la concentration sur les citoyens}

La concentration sur le citoyen est utile pour impliquer l'institution publique dans un change, procès qui suppose le transfère volontaire d'un produit (service public, prestation publique) vers un client, en échange d'un coût (financier ou non financier) supporté par celui-ci. L'inspection sociale a la tâche de contrôler ce produit ayant dans sa pensée "La théorie de l'échange», selon laquelle ce que nous offrons au public, le but (les bénéfices) doit être considéré au moins égal avec ce qu'elle devra offrir (les coûts). Pour élaborer des stratégies orientées vers les citoyens, on a besoin d'informations (des études de marché, le cas dans la situation donnée est l'évaluation du système social), qui doit donner des réponses claires aux questions fondamentales. Les coûts peuvent avoir ou non un caractère pécuniaire et les obstacles rencontrés peuvent être extrêmement variés, de la non reconnaissance de l'existence d'un produit (service public, prestation publique) jusqu'à la perception d'un manque d'aptitudes nécessaires pour l'adoption d'un comportement adéquat de l'employé public. Quels bénéfices s'imaginent les potentiels clients obtenir (par exemple un citoyen qui se présente à la Commission d'évaluation des personnes avec handicap) et quels sont les plus appréciés! Par exemple quel est le plus grand bénéfice perçu par un citoyen pour le fait qu'il est testé HIV/SIDA? Il s'agit d'une prolongation de la vie à cause du commencement au bon moment du traitement? Il s'agit de la paix de l'âme? Ou il peut s'agir de la capacité de s'assumer la responsabilité de ne pas repentir la maladie?

Le processus de prise de la décision par le citoyen est formé, selon la théorie de cinq étapes: on exemplifie l'achat d'un service: I'hospitalisation dans un centre de Soin et Assistance: 
* La reconnaissance du besoin d'hospitalisation (généralement, des personnes âgées, qui, soufrant d'une déficience fonctionnelle font appel à un tel service):

* La recherche d'informations (la famille, les connaissances ou même la personne en cause doivent être informés en ce qui concerne l'existence de tels centres, aux paquets de services minimales, aux standards etc.)

* L'évaluation des variantes (centres publics, privés, en partenariat public privé):

* La décision d'achat du service (l'avancement du dossier personnel de hospitalisation vers l'autorité compétente en vue d'être résolue, respectivement le conseil local ou départemental);

* Le comportement post-acquisition (la satisfaction du client)

Dans la réalité de tous les jours de la Roumanie, le citoyen peut passer directement à la reconnaissance du besoin d'acquisition d'un service ou, il ne peut jamais arriver dans la posture d'acheteur.

Principe 2 : la segmentation et le choix des piste cible

\section{Retour à la table des matières}

Les marchés sont des groupes de citoyens existants et potentiels (par ex. les personnes avec un handicap bénéficiaires de la gratuité du transport urbain), L'une des prémisses fondamentales est celle selon laquelle ils différent, la plupart des cas, les uns des autres au moins d'un point de vue. Ils peuvent être différents le plus probablement à cause du handicap (locomoteur, somatique, sourdité - cécité, aveugles etc.), mais aussi du point de vue des valeurs, attitudes, ressources, situations géographiques ou même des expériences antérieures eues avec l'administration publique.

Une possible variante de segmentation est celle connue sous le nom de La Théorie de la diffusion des innovations, selon laquelle les gens différent entre eux selon la disponibilité d'essayer de nouveaux produits et services. La segmentation du marché est faite comme il suit: 
1. Les innovateurs - sont les plus audacieux et ils acceptent aisément les nouvelles idées:

2. Les acceptants précoces - ils sont motivés par les leaders d'opinion et ils adoptent plus tôt des nouvelles idées, mais ils sont vigilants;

3. La majorité précoce - elle hésite quand il s'agit d'adopter un produit ou un service nouveau, actionnant avant les personnes habituelles:

4. La majorité retardée - elle est sceptique et elle adopte un produit ou un service nouveau seulement quand la majorité des gens l'ont essayé déjà;

5. Les retardés - ils sont suspicieux vers les changements et ils les acceptent seulement quand ils sont devenus tradition ou coutume.

En réalité, l'administration publique centrale et locale de la Roumanie rend service aux marchés complexes. Pour l'Inspection Sociale il est important et recommandé de savoir quand le marché est diversifié et il a besoin d'être abordé différemment (par exemple dans le cas des besoins de services spécialisés de récupération pour une certaine catégorie de personnes avec handicap, présentes dans un pourcentage significatif dans la zone géographique respective).

Principe 3 : l'identification de la concurrence

\section{Retour à la table des matières}

L'identification de la concurrence, surnommée aussi la myopie de marketing ${ }^{5}$, dans des termes très stricts, les concurrents peuvent être définis comme des organisations qui offrent aux mêmes clients des produits et des services semblables avec ceux offerts par les institutions publiques, souvent à des prix similaires 6 .

5 Article publié en Harvard Business Review 1960-par le prof. Theodore Levitt-qui affirme que les sociétés de transport sur la voie ferrée ont provoqué seul leur déclin en insistant sur l'idée que «notre domaine d'activité est la voie ferrée». S'il avait dit que le domaine d'activité est le transport, il aurait observé la concurrence, qui fait son apparition automobiles, camions, avions. Le besoin était de transport- en général - et non de transport sur la voie ferrée.

6 Romhandicap - institution de prothèses subventionnée par le Gouvernement de la Roumanie a perdu le marché, à cause du management inadéquat disparaissant, après 17 ans d'activité, du marché interne et étant remplacé par des organisations non gouvernementales compétitive sur le marché des prothèses et orthèses pour des personnes avec handicap. 
Il y a 5 stratagèmes vérifiés pour conquérir et garder la position vedette sur tout marché 7 :

* Le stratagème de la course de vitesse-le plus simple stratagème, qui suppose la lutte directe avec un concurrent et il se concentre sur le « dépassement » de celui-ci :

* Le stratagème de la plate-forme-on attire de l'Inspection Sociale d'autres organisations pour lutter contre les concurrents communs (par ex. I'association avec les organisations professionnelles comme, par exemple, le Collège des Psychologues de Roumanie, le Collège des Assistants Sociaux de Roumanie, I'Association des Aveugles de Roumanie, etc.) :

* Le stratagème de l'intrusion - survivance en évitant la confrontation directe (par ex. Romhandicap qui a décidé de ne pas entrer en concours avec d'autres firmes de prothèses - mais ce stratagème n'a pas fonctionné à long terme) :

* Le stratagème du meilleur des deux-on suit la ligne moyenne, entre deux variantes opposées (ex. Rosal prothèse s'adresse à ceux intéressés de prothèses roumaines ainsi qu'à ceux intéressés de prothèses ultra performantes d'importation):

* Le stratagème "haut bas »-offre une variante chère et une variante bon prix (ex. Les Conseils départementaux et locaux offrent des services de soin et d'assistance pour les personnes âgées à des paquets minimaux, qui peuvent être couverts d'une pension moyenne, ou des services spécialisés à des prix de jusqu'au 1500 EUR/mois).

7 The Marketing Playbook-John Zagula et Richard Tong - anciens dirigeants du Microsoft, offre une perspective claire, pratique sur les stratégies de concurrence. 
Principe 4 : l'utilisation des 4 instruments du mixe de marketing - le produit, le prix, la distribution, la promotion

\section{Retour à la table des matières}

Ce principe dit que le marketing est plus que la plupart des gens le croient quand ils entendent ou utilisent ce terme. Dans les scénarios idéaux en ce qui concerne la planification des activités de marketing, les décisions concernant la promotion sont mises en question quand le produit, le prix et la distribution se sont précisés avec exactitude. Ex. :

Produit = la subvention pour la chaleur

Prix = le coût que les conseils locaux par les directions générales d'assistance sociale le payent aux personnes habilitées par loi de le recevoir

La distribution = prévoit le lieu, le moment et la modalité par lesquels les citoyens sont habilités de recevoir cette prestation sociale

La promotion = communication à caractère persuasif (par rapport à celles avec un caractère informatif ou éducatif), et la stratégie de communication comprend une combinaison de publicité, présentations publiques et marketing directe. Le vrai objectif de la promotion est la transmission d'un message et l'assurance que le public visé apprend de l'offre et il recevra les bénéfices dont on parle et il est déterminé d'agir.

\section{Principe 5 : la suite de l'activité et l'ajustement}

Beaucoup d'appels viennent de la société civile vers les institutions publiques, il s'agit même des demandes d'augmenter l'efficacité en vue de l'amélioration de l'activité. C'est ainsi que les institutions publiques doivent déclencher un processus de contrôle destiné à maximiser la probabilité que l'organisation atteigne les objectifs établis par court, moyen et long terme. La première composante essentielle du système représente la compréhension parfaite des objectifs. Il doit ensuite disposer d'instruments avec lesquels il va mesurer les résultats. Il faut ensuite en faire une analyse minutieuse ainsi que l'identification des pas faits pour réaliser les corrections qui s'imposent. 
Le processus de contrôle 8

* Établir les objectifs

* Qu'est-ce quion veut réaliser?

* Mesurer les résultats

* Que se passe-t-il ?

* Établir le diagnostic

* Pourquoi arrive-t-il ?

* Mesures correctives

* Qu'est-ce qu'il faut faire?

\section{La création de l'Inspection Sociale de la Roumanie}

Retour à la table des matières

C'est à la pression de l'Union Européenne qu'on a créé l'Inspection Sociale par O.U.G. no. 130/2006 du 30 décembre 2006 approuvée par la Loi no.211/2007, qui a comme but le contrôle de l'implémentation de la législation dans le domaine, ainsi que l'inspection de l'activité des institutions publiques et privées, responsables avec la livraison des prestations et des services sociales. A la fin de l'année 2007 et dans les premiers mois de l'année 2008 mass média de la Roumanie et de l'étranger assume la responsabilité de plusieurs problèmes sociaux qui se sont empirés (par ex. le Traitement de l'enfant soigné par l'assistant maternelle de la province, la crise du système de récupération et de réhabilitation des personnes avec un handicap neuropsychique des centres de psychiatrie, la croissance du numéros des personnes avec un handicap et l'inefficacité des commissions d'évaluation de celles-ci etc.). Le besoin de contrôle, d'évaluation et de conseil du domaine social se ressentent par l'apparition d'une nouvelle institution habiletée par MMFES, Inspection Sociale, avec des objectifs concrets (jusqu'à la modification de la législation dans le domaine), et avec les meilleurs partenaires potentiels (les citoyens de la Roumanie). L'Inspection Sociale a démarré par un projet de twining light en collaboration avec l'Inspection Sociale de la France, étant traitée avec ajustements et appliqué, le modèle français. Le produit, respectivement l'Inspection Sociale, comme terme utilisé dans le secteur public, n'est pas encore familial mais ce terme a plusieurs interprétations et il se

8 Andreasen and Kotler, strategic Marketing, 2008. 
rapporte à tout service ou prestation publique qui est offert sur le marché de la Roumanie par l'organisation (soit elle publique ou privée), pour satisfaire une demande ou un besoin. Il comprend les biens matériels et les services sociaux, mais il se rapporte aussi à d'autres choses que l'organisation « vend», comme par exemple des événements, gens - employés publics, informations, idées, etc.

Ex. La gamme de produits de l'Inspection Sociale est présenté dans le tableau suivant.

\begin{tabular}{|c|c|}
\hline Le type de produit & Exemples de produits \\
\hline $\begin{array}{l}\text { Des biens matéri- } \\
\text { aux }\end{array}$ & $\begin{array}{l}\text { Le registre électronique des fournisseurs de services sociaux } \\
\text { de la Roumanie } 9\end{array}$ \\
\hline Programmes & $\begin{array}{l}\text { Programme d'évaluation de l'état de fait des institutions pu- } \\
\text { bliques d'assistance sociale }\end{array}$ \\
\hline Services & $\begin{array}{l}\text { L'Analyse et la diagnose de la rémunération (indemnisation) de } \\
\text { l'assistant personnel de la personne avec un handicap, qui inclue } \\
\text { l'assistant personnel professionnel, ainsi que les conditions de } \\
\text { soin au domicile }\end{array}$ \\
\hline Evénements & $\begin{array}{l}\text { Rencontres avec les associés professionnelles et ONG avec un } \\
\text { objet d'activité dans le domaine social }\end{array}$ \\
\hline Gens & La formation de l'inspecteur social \\
\hline Lieux & Les 8 sièges régionaux de l'Inspection Sociale \\
\hline Organisations & $\begin{array}{l}\text { Les directions générales d'assistance sociale et protection de } \\
\text { l'enfant }\end{array}$ \\
\hline Informations & Le guide de l'inspecteur social \\
\hline Idées & $\begin{array}{l}\text { Protocole achevé avec la Police routière en vue de donner des } \\
\text { amendes aux possesseurs d'auto véhicules qui sont parqués sur } \\
\text { les lieux destinés aux personnes avec un handicap }\end{array}$ \\
\hline
\end{tabular}

9 Il y a une base de données prise de ANPH, ANPDC et DGASPC, mais le registre unique n'a pas été délivré jusqu'à présent 
Le plus important fait est celui que les bénéficiaires de l'Inspection Sociale ont une très grande variété de besoins et d'aspirations, et la plupart considère qu'il est peu probable qu'ils soient contents avec une seule offre, ou même avec un seul type d'offre.

Le changement du modèle de management des institutions publiques est inévitable et elle va produire des effets sur les citoyens ainsi que sur le secteur privé. L'existence ou le niveau du financement n'est pas garanti aux institutions publiques. Elles devront comprendre, elles aussi, la dynamique des technologies qui sont en changement. Chaque institution publique devra avoir une conception stratégique propre, avoir en vue de nouvelles pratiques, efficaces; se renouveler, innover et savoir présenter les mérites par des stratégies d'image très clairement conçues.

\section{Bibliographie}

Retour à la table des matières

1. Academia Română, Institutul Național de Cercetări Economice, Calitatea Vieții , 1997-www.iccv.ro

2. Andreasen and Kotler - Strategic Marketing, Teora, Bucuresti, 2008 (editie revizuita)

3. Anuarul Statistic al României, Comisia Națională de Statistică, 2008

4. D. Autissier, J. Moutot, Méthode de conduite du changement, Collection : Stratégies et Management, Editura Dunod, Paris, ISBN10 : 2-10-051062-2, 14.11.2007.

5. David Autissier, Jean Michel Moutot, L'entreprise en movement-Conduite et mise en oeuvre du changement: l'effet de levier, Paris, Editura Dunod, 2008.

6. Barton Laurence, Crisis in Organisation: Managing and Communicating in the Heat of Chaos, South Westwrn Publishing, Cincinatti, 1993

7. Barus-Michel Jacqueline, Giust-Despaires Florence, Ridel Luc, Crize, Abordarea psihosociologică clinică. Editura Polirom, Iaşi, 1998

8. W.G. Bennis, Diriger. Les secrets des meilleurs leaders, InterEditions, Paris, 1985

9. Warren G. Bennis, Kenneth D. Benne, and Robert Chin, The Planning of Change (2nd Edition). (Eds.). Holt, Rinehart and Winston, New York: 1969. 
10. Berger, Hilary - Agile development in a bureaucratic arena - A case study experience, International Journal of Information Management, 2007

11. Boorstin Daniel I., The image. A guide to Pseudo-Events în America.. New York, SUA, Atheneum, 1985.

12. Boudon Raymond, Tratat de sociologie, Ed. Humanitas, Bucureşti, 2007.

13. Brodi E.W., Managing Communication Processes, From Planning to Crisis Response. Praeger, New York,1991.

14. Bulletin Officiel du Travail, de l'Emploi et de la Formation Professionnelle $n^{\circ}$ 2002/16 du jeudi 5.09.2002 /Apprentissage/ Ministère des Affaires Sociales, Du Travail Et De La Solidarité, Délégation générale à l'emploi et à la formation professionnelle, Sous-direction des formations en alternance et de l'insertion des jeunes

15. Cavagnol, Pascal Roulle, Management des organizations, Editura Gualino, Paris, ISBN 978-2-297-01021-4, 10.02.2009.

16. Circulaire DGEFP $n^{\circ} 2002-37 d u 19$ juillet 2002 concernant la mise en œuvre de la procédure d'urgence dans le cadre de l'apprentissage et la résiliation du contrat d'apprentissage

17. Coyne, J. Christopher - The Politics of Bureaucracy and the failure of postwar reconstruction, Springer, Science+Business Media, LLC, 2007.

18. Ferrary Michel, Pesqueux Yvon, Management de la Connaissance et la société de la connaissance, Editura Economique, Artois, 2006.

19. Galenson Walter, The Historical Role of American Trade Unionism, Institute for Contemporary Studies, San Francisco, California, 1986

20. Gilles Ferreol, Identitatea, cetățenia şi legă turile sociale, Ed. Polirom,2000

21. Grant Wendy, Rezolvarea conflictelor, Ed. Teora, București, 1997.

22. Hall Richard, Organizations. Structures, Processes and Outcomes, PrenticeHall International. Inc., 1996).

23. Harrison, M.I.; Shiron, A., (1999) - Organizational diagnosis and assessment, Bridging theory and practice, Sage Publications, Londra.

24. Johns Gary, Comportament organizațional, Ed. Economica, Bucureşti, 2002.

25. Gh.Ionescu, A.Toma, Cultura organizațională şi managementul tranzịtiei, Ed. Economică, București, 2001

26. Macy, B.A.; Izumi,H. (1993) - "Organizational change, design and work innovations; A meta-analysis of 131 North American field studies, 1961-1991"- Research in Organizational Change and Development, nr.7, pag.235-313

27. McLonglin Barry, Risk and Crissis Communication, Mc.Longlin Multimedia Publishing Ltd., Ottawa, 1996. 
28. Mucchielli R., La dynamique des groupes, Les Editions Sociales, Francaises, Paris, 1970.

29. Newby, T.J., Corner, j. (1997) - Mentoring for increased performance : fondations \&methods, $n r .36$ (2), pag.11-15.

30. Pauchant Thierry C., Transforming the Crisis-Prone Organizations. San Francisco, California, Jossey-Boss, 1992.

31. Pinsdorf Marion K., Communicating when Your Company is under Siege; Surviving Public Crisis, Lexington, Massachusetts, 1987

32. Prigogine Ilya - Self organization in nonequilibrium systems, New York, Wiley end Sons, 1977

33. Evaluarea starii de fapt a domeniului social - 2002" - proiect national de cercetare sociologica, derulat de Inspectia Regionala Bucuresti - MMPS (manager proiect, Iustina Radu-inspector sef al I.R.Bucuresti), 2003.

34. "Riscuri de securitate in regiunea Marii Negre - analiza geopolitica si geostrategica a fenomenului handicapului din Romanid" proiect initiat si coordonat de Iustina Radu, in cadrul Institutului Diplomatic Roman - 2006-2007 - si finalizat in lucrarea de licenta "Riscuri de securitate in regiunea Marii Negre" - lucrare publicata in Revista Institutului Diplomatic Roman, 2007)

35. Suport de curs - "Curs de formare profesionala a inspectorului social Fundamentele metodologice şi epistemologice ale asistenței sociale" acreditat de CNFPA-MMPSF, redactat de M.Anton si Iustina Radu, Bucuresti, 2008

36. Suport de curs - "Curs de formare profesionala a inspectorului social - Managementul resurselor umane în domeniul social" acreditat de CNFPA- MMPSF, redactat de T.Borcea si Iustina Radu, Bucuresti, 2008

37. "Elaborarea standardului ocupational si formarea asistentului personal al persoanei cu handicap - 2005-2006" - proiect national derulat de Inspectia Regionala Bucuresti in parteneriat cu Centrul National de Resurse Juridice si Institutul National de Combatere a Excluziunii Sociale (Iustina Radu - coordonator proiect formare profesionala asistent personal al persoanei cu handicap)

38. Iustina Radu - Riscuri de securitate in regiunea Marii Negre, Revista Institutului Diplomatic Roman, octombrie 2007.

39. Iustina Radu - "Managementul schimbarii in organizatii" (articol) publicat in Buletinul Universitatii Nationale de Aparare "Carol I", nr.3, 2008, publicație ştiințifică de importanță națională de categoria "B+" recunoscută de Consiliul Național al Cercetării Stiințifice din Invățământul Superior (CNCSIS), Editura Universității Naționale de Apărare "CAROL I" Bucureşti - 2008, ISSN 1584 1928.

40. Raymond, Efecte perverse şi ordine socială, Polirom, 1993 
41. Revue d'Evaluation Transversale Et Thématique, Ministère - direction de la sécurité sociale, CPAM de Strasbourg, CAF de la Moselle, CMR Alsace, ORGANIC Lorraine, 18 mars 2000

42. Revue de l'inspection sociale et le droit pénal social, nr.10, 2002, Tout savoir sur les sanction du travail en noir, de l'occupation de travailleurs clandestins et sur l'attitude à adopter vis-à-vis de l'Inspection sociale, Ministère des Affaires Sociales.(Belgique)

43. Linda Shaw, Medierea conflictelor comunitare.. Mediere-Nivel I; MediereNivel II, suport de curs al Centrului de mediere şi securitate comunitară-Iaşi

44. Edouard Stacke, Coaching d'entreprise, performance et humanisme, Editura Broche, Editia a II-a, Paris, 2006.

45. Stanton Nick, Comunicarea, Ed. Societatea Ştiință şi Tehnică S.A., Bucureşti, 1995.

46. Stephen Robbins, Organizational Behaviour, Concepts, Controversies, Applications, Prentice - Hall, Inc.1998.

47. Rene Thom - Parabole et catastrophe, Paris, Vrin, 1972.

48. James D. Thompson. McGraw-Hill, Organizations in Action. New York: 1967.

49. Verboncu Ion, Management şi manageri, Ed.Economică, Bucureşti,2001.

50. Vlăsceanu Mihaela, Organizații şi comportament organizațional,Ed.Polirom, 2003.

51. Watzlawick Beavin, Une logique de la communication, Edition du Seuil, Paris, 1972

52. Sistemele de protecție sociala din țările UE (MISSOC): http://ec.europa.eu/employment_social/spsi/missoc_en.htm

53. Guvernul României www.guv.ro

54. European Centre for Social Welfare Policy and Research (Vienna): http://www.euro.centre.org

55. The World Wide Evaluation Information Gateway (social policy evaluations): http://www.policy-evaluation.org

56. Observatoire Social Europeen: http://www.ose.be/en/default.htm

57. Office quebecois pour les personnes handicapees (interventions/adaptations) www.ophq.gouv.qc.ca

58 http://socialsecurity.fgov.be.fr Entretien avec Pol C., inspecteur social

\section{Fin du texte}

